\title{
Experience of malignancies with oral glucose-lowering drugs in the randomised controlled ADOPT (A Diabetes Outcome Progression Trial) and RECORD (Rosiglitazone Evaluated for Cardiovascular Outcomes and Regulation of Glycaemia in Diabetes) clinical trials
}

\author{
P. D. Home • S. E. Kahn • N. P. Jones $\cdot$ D. Noronha $・$ \\ H. Beck-Nielsen • G. Viberti • \\ for the ADOPT Study Group and the RECORD \\ Steering Committee
}

Received: 23 August 2009/Accepted: 12 April 2010/Published online: 8 June 2010

(C) The Author(s) 2010. This article is published with open access at Springerlink.com

\begin{abstract}
Aims/hypothesis Observational and mechanistic studies have suggested a possible relationship between treatment with metformin and decreased incidence of cancer in participants with type 2 diabetes. We extracted data for malignancies from the ADOPT (A Diabetes Outcome
\end{abstract}

P. D. Home $(\bowtie)$

ICM - Diabetes, The Medical School, Newcastle University,

Framlington Place,

Newcastle upon Tyne NE2 4HH, UK

e-mail: philip.home@newcastle.ac.uk

\section{S. E. Kahn}

Division of Metabolism, Endocrinology and Nutrition,

Department of Medicine,

Veterans Affairs Puget Sound Health Care System and University

of Washington,

Seattle, WA, USA

N. P. Jones

GlaxoSmithKline Research \& Development,

Harlow, UK

\section{Noronha}

GlaxoSmithKline Research \& Development,

Greenford, UK

H. Beck-Nielsen

Endocrinology Research Unit, University of South Denmark,

Odense, Denmark

\section{G. Viberti}

Unit for Metabolic Medicine,

King's College London School of Medicine,

London, UK
Progression Trial) and RECORD (Rosiglitazone Evaluated for Cardiovascular Outcomes and Regulation of Glycaemia in Diabetes) randomised controlled clinical trials, in which the efficacy and/or safety of metformin was assessed in comparison with sulfonylureas and rosiglitazone.

Methods Neoplasm occurrences were collected as adverse events in these studies. We reviewed and re-analysed the individual participant data in both studies for serious adverse events, malignancies reported as adverse events and related neoplasms of special interest.

Results In ADOPT, 50 participants (3.4\%) on metformin and $55(3.8 \%)$ on each of rosiglitazone and glibenclamide (known as glyburide in the USA and Canada) developed serious adverse event malignancies (excluding nonmelanoma skin cancers). This corresponds to $1.03,1.12$ and 1.31 per 100 person-years, giving hazard ratios for metformin of $0.92(95 \%$ CI $0.63-1.35)$ vs rosiglitazone and $0.78(0.53-1.14)$ vs glibenclamide. In RECORD, on a background of sulfonylurea, 69 (6.1\%) participants developed malignant neoplasms in the metformin group, compared with $56(5.1 \%)$ in the rosiglitazone group (HR 1.22 [0.86-1.74]). On a background of metformin, $74(6.7 \%)$ participants in the sulfonylurea group developed malignant neoplasms, compared with $57(5.1 \%)$ in the rosiglitazone group (HR 1.33 [0.94-1.88]).

Conclusions/interpretation The malignancy rates in these two randomised controlled clinical trials do not support a view that metformin offers any particular protection against malignancy compared with rosiglitazone. However, they do not refute the possibility of a difference compared with sulfonylureas. 
Keywords Cancer - Diabetes mellitus · Metformin . Rosiglitazone $\cdot$ Sulfonylureas $\cdot$ Thiazolidinediones

$\begin{array}{ll}\text { Abbreviations } \\ \text { ADOPT } & \text { A Diabetes Outcome Progression Trial } \\ \text { RECORD } & \begin{array}{l}\text { Rosiglitazone Evaluated for Cardiovascular } \\ \text { Outcomes and Regulation of Glycaemia in } \\ \text { Diabetes }\end{array} \\ \text { UKPDS } & \text { UK Prospective Diabetes Study }\end{array}$

\section{Introduction}

Observational analyses of data from population registries that include people with diabetes have suggested an association between use of metformin and decreased prevalence of malignancy [1-3]. Knowledge of the cellular biochemical effects of metformin suggests a putative mechanism by which such an effect could be mediated $[4,5]$. Together the above notions have resulted in metformin being endorsed as good news for people with diabetes [6].

Observational studies are subject to various recognised kinds of bias. Metformin may be a particular problem here, as prescribers respond variably to concern in the elderly, and in patients with renal, hepatic and cardiac disease, targeting it to the obese but otherwise healthy individual [7]. Mechanistic studies may also suffer attribution bias. Randomised controlled clinical trials are generally believed to offer a higher level of evidence, although they, too, are not without problems. Here, we have taken the opportunity offered by two large, long-term studies in people with type 2 diabetes, in which metformin was randomised and compared with other oral glucose-lowering agents, in order to determine malignancy rates in participants on these medications $[8,9]$.

\section{Methods}

All sponsored clinical trials are required to routinely report serious adverse events to the manufacturer. The International Conference on Harmonisation of Technical Requirements for Registration of Pharmaceuticals for Human Use recommends that cancers should be characterised as 'medically important' adverse events and therefore classified as serious adverse events [10], although some skin cancers may only be reported as adverse events. Sponsor manufacturers maintain comprehensive databases of serious adverse events and malignancies, and routinely prepare clinical trial reports with a major focus on adverse events soon after completion of each study. For the current report, the individual participant data of the following two studies were accessed and examined: (1) A Diabetes Outcome Progression Trial (ADOPT; ClinicalTrials.gov registration no. NCT00279045); and (2) Rosiglitazone Evaluated for Cardiovascular Outcomes and Regulation of Glycaemia in Diabetes (RECORD; ClinicalTrials.gov registration no. NCT00379769).

Manufacturer-sponsored studies, including ADOPT and RECORD, are subject to site monitoring by external staff to ensure study conduct. One activity of monitors is to ensure local investigators identify and report serious adverse events faithfully. However, some adverse events occur remote from the study site and full medical details may not be available. Where a term, such as 'breast neoplasm' is ambiguous as to malignancy and no further information can be obtained, it is counted as malignancy. In the summary tables presented here, lower level terms referring to one organ site (e.g. 'colon cancer', 'colorectal carcinoma', 'malignant neoplasm of sigmoid colon', 'adenocarcinoma of colon') have been grouped together. Adverse events were assessed for malignancies without reference to treatment allocation.

Data are presented as participants with a malignancy (reported as a serious adverse event, but excluding nonmelanoma skin cancers unless explicitly indicated otherwise), per cent of those exposed and rate per 100 person-years. Kaplan-Meier curves are presented to provide visual timedependent analysis. Hazard ratios and 95\% CIs were calculated by Cox proportional hazards regression for metformin vs randomised comparators in the two studies and for sulfonylureas vs rosiglitazone in the RECORD study. Multivariate analysis to take account of crossover and other drug usage was not relevant to ADOPT and was not attempted for the RECORD study because of the difficulties in identifying likely bias arising from post-randomisation treatment allocation [11].

Role of the study sponsor The sponsor of the studies analysed (GlaxoSmithKline) archived the study data and assisted in analysis of the data used in the present report. Some further background data on the RECORD study was supplied by sponsor statisticians. Otherwise, the paper was prepared entirely by the authors, one of whom is an employee of GlaxoSmithKline who is engaged in both source studies, and one of whom is a company statistician at GlaxoSmithKline.

\section{Results}

ADOPT clinical trial ADOPT was a monotherapy study of efficacy of glucose-lowering with double-blind randomisation to metformin, rosiglitazone and glibenclamide 
Table 1 Patient exposure and malignancies in ADOPT

Data are based on number $(n)$ of patients; malignancies are those reported as serious adverse events excluding non-melanoma skin cancers

${ }^{a}$ Adjusted for study exposure

\begin{tabular}{llll}
\hline Variable & Metformin & Rosiglitazone & Glibenclamide \\
\hline$n$ & 1,454 & 1,456 & 1,441 \\
Study exposure (person-years) & 4,906 & 4,954 & 4,244 \\
Malignancies, $n(\%)_{\text {Rate (per 100-person-years) }}{ }^{\mathrm{a}}$ & $50(3.4)$ & $55(3.8)$ & $55(3.8)$ \\
HR $(95 \% \mathrm{CI})$ vs rosiglitazone & 1.03 & 1.12 & \\
HR $(95 \% \mathrm{CI})$ vs glibenclamide & $0.92(0.63-1.35)$ & & \\
\hline
\end{tabular}

(known as glyburide in the USA and Canada) [8]. Median duration in the study was 4.0 years. Numbers treated were very similar in the three groups, being 1,454, 1,456 and 1,441 respectively, with exposure of 4,906, 4,954 and 4,244 person-years respectively. Monotherapy failure occurred by study end in $21 \%$ participants on metformin, $15 \%$ on rosiglitazone and $34 \%$ on glibenclamide; participants were not followed beyond this endpoint.

Malignancies reported as serious adverse events, but excluding non-melanoma skin malignancies, occurred in 50 participants $(3.4 \%)$ on metformin and in $55(3.8 \%)$ on each of rosiglitazone and glibenclamide. This corresponded to 1.03, 1.12 and 1.31 malignancies per 100 patient-years (Table 1). The hazard ratio for metformin compared with rosiglitazone was $0.92(95 \%$ CI $0.63-1.35)$ and that for metformin vs glibenclamide was 0.78 (95\% CI 0.53-1.14) (Table 1). Kaplan-Meier cumulative event curves are given in Fig. 1.

RECORD clinical trial RECORD was a 5.5 year cardiovascular outcomes study of oral glucose-lowering combination therapy with rosiglitazone or metformin added to sulfonylurea in one stratum, and rosiglitazone or sulfonylurea added to metformin in the other stratum [9]. Accordingly, randomised comparisons can only be made between metformin and rosiglitazone, and between sulfonylurea and rosiglitazone within strata, but not between metformin and sulfonylurea.

On background sulfonylurea, 1,122 people were randomised to metformin and 1,103 to rosiglitazone. Total study exposure was 6,126 and 6,110 person-years respectively, with $82 \%$ of this time including exposure to metformin and $87 \%$ to rosiglitazone, but only $9.5 \%$ and $5.4 \%$ respectively including exposure to insulin. As a result of a rescue therapy algorithm, 27\% of those in the rosiglitazone group also had some exposure to metformin later in the study.

Malignancies reported as serious adverse events, but excluding non-melanoma skin malignancies, occurred in $69(6.1 \%)$ participants in the metformin group, compared with $56(5.1 \%)$ participants in the rosiglitazone group, giving a hazard ratio of 1.22 (95\% CI $0.86-1.74)$ (Table 2). Kaplan-Meier cumulative event curves are given in Fig. 1. Per protocol (while on the randomised dual therapy), 54 (4.8\%) participants developed malignancy on metformin and $43(3.9 \%)$ on rosiglitazone (HR 1.12 [95\% CI 0.75-1.68]).

On background metformin, 1,105 people were randomised to sulfonylurea and 1,117 to rosiglitazone. The people in this stratum were notably more obese, but had shorter duration of diabetes than those on background sulfonylurea [9]. Total study exposure to sulfonylurea and rosiglitazone was 6,146 and 6,228 person-years respectively, with $84 \%$ of this time including exposure to sulfonylurea and $88 \%$ exposure to rosiglitazone, but only $10 \%$ and $4 \%$ of the time respectively including exposure to insulin. As a result of a rescue therapy algorithm, 23\% of those in the rosiglitazone group also had some exposure to sulfonylurea later in the study.

In the group randomised to sulfonylurea, $74(6.7 \%)$ participants developed malignant neoplasm, compared with $57(5.1 \%)$ in the rosiglitazone group, hazard ratio 1.33 (95\% CI 0.94-1.88). Kaplan-Meier cumulative event curves are given in Fig. 1. Per protocol (while on the
Table 2 Patient exposure and malignancies in RECORD

Data are based on number $(n)$ of patients; malignancies are those reported as serious adverse events excluding non-melanoma skin cancers

${ }^{\text {a }}$ Adjusted for study exposure

\begin{tabular}{llllll}
\hline Variable & \multicolumn{2}{l}{ Background sulfonylurea } & & \multicolumn{2}{l}{ Background metformin } \\
\cline { 2 - 3 } \cline { 5 - 6 } & Metformin & Rosiglitazone & & Sulfonylurea & Rosiglitazone \\
\hline$n$ & 1,122 & 1,103 & & 1,105 & 1,117 \\
Study exposure (person-years) & 6,126 & 6,110 & & 6,146 & 6,228 \\
Malignancies, $n(\%)$ & $69(6.1)$ & $56(5.1)$ & & $74(6.7)$ & $57(5.1)$ \\
Rate (per 100 person-years) & 1.15 & 0.94 & & 1.23 & 0.93 \\
HR (95\% CI) & $1.22(0.86-1.74)$ & & & $1.33(0.94-1.88)$ & \\
\hline
\end{tabular}


Fig. 1 Kaplan-Meier event curves for serious adverse event malignancies in (a) ADOPT, and (b) the metformin stratum and (c) the sulfonylurea stratum of RECORD. Hazard ratios are given in Tables 1 and 2. Numbers below panels, participants (n) evaluable each year. Circles, metformin; squares, rosiglitazone; diamonds, sulfonylurea
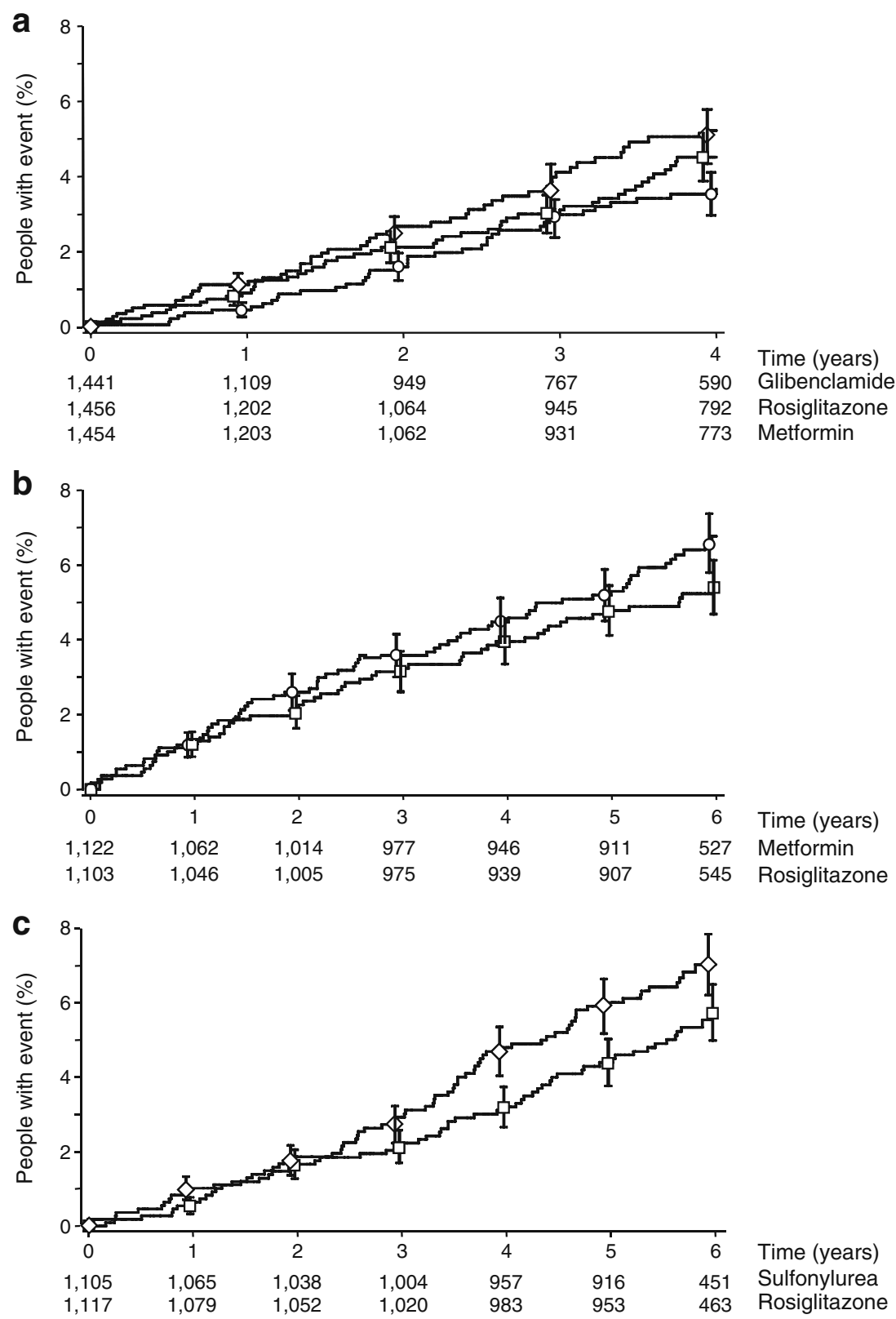

randomised dual therapy), 60 (5.4\%) participants developed malignancies on sulfonylurea and $44(3.9 \%)$ on rosiglitazone (HR 1.25 [95\% CI 0.85-1.18]).

Single-organ category analyses In the ADOPT study the number of malignancies reported in any one organ category was low (Table 3). Some imbalance in non-melanoma skin malignancies was noted. For men, prostate cancer rates did not appear to differ between medications. For women, breast, ovarian and uterine cancers showed no consistent trends. No additional non-serious adverse event malignancies (except non-melanoma skin) were identified.

Few malignancies in any one organ category were reported in the RECORD study (Table 4). The only malignancy group with a possible trend in favour of metformin was skin cancers (three vs eight cases). Additionally, one case of prostatic carcinoma in a participant allocated to rosiglitazone was reported as a non-serious adverse event. Overall, prostate cancer rates in men did not appear to differ between medications. In women, breast, ovarian and uterine cancers showed no consistent trends (Table 4).

Combined study/strata analysis No formal multiple treatment comparison between strata in the RECORD study has been performed, but on the assumption of equivalence for rosiglitazone (Table 2), the relative risk for metformin vs sulfonylurea would be 0.92 . The ADOPT data comparing metformin with rosiglitazone could be combined (meta- 
Table 3 Numbers of participants developing malignancies in ADOPT
Data are number $(n)$ of patients (\%) with a neoplasm reported as a serious adverse event

Per cent values for prostate and for breast, cervix/uterine and ovarian cancer are based on men only and women only

\begin{tabular}{|c|c|c|c|}
\hline Variable & Metformin & Rosiglitazone & Glibenclamide \\
\hline$n$ & 1,454 & 1,456 & 1,441 \\
\hline Study exposure (person-years) & 4,906 & 4,954 & 4,244 \\
\hline \multicolumn{4}{|l|}{ Malignancies } \\
\hline All & $56(3.9)$ & $60(4.1)$ & $64(4.4)$ \\
\hline All non-skin & $50(3.4)$ & $55(3.8)$ & $55(3.8)$ \\
\hline Breast & $6(1.0)$ & $6(0.9)$ & $3(0.5)$ \\
\hline Endocrine & 0 & 0 & $2(0.1)$ \\
\hline Colorectal & $7(0.5)$ & $4(0.3)$ & $10(0.7)$ \\
\hline Gastric & $1(0.1)$ & $1(0.1)$ & $1(0.1)$ \\
\hline Hepato-biliary & 0 & $3(0.2)$ & $1(0.1)$ \\
\hline Intestinal & $1(0.1)$ & $1(0.1)$ & 0 \\
\hline Oral and oesophagus & $2(0.1)$ & $3(0.2)$ & $3(0.2)$ \\
\hline Pancreatic & 0 & $5(0.3)$ & 0 \\
\hline Haematological & $3(0.2)$ & $4(0.3)$ & $4(0.3)$ \\
\hline Metastases unknown & 0 & $2(0.1)$ & $2(0.1)$ \\
\hline Unspecified & $2(0.1)$ & $3(0.2)$ & $1(0.1)$ \\
\hline Nervous system & $2(0.1)$ & 0 & 0 \\
\hline Bladder & $4(0.3)$ & $2(0.1)$ & $4(0.3)$ \\
\hline Renal & $1(0.1)$ & $2(0.1)$ & $2(0.1)$ \\
\hline Cervix/uterine & $1(0.2)$ & $1(0.2)$ & $3(0.5)$ \\
\hline Ovarian & 0 & $3(0.5)$ & 0 \\
\hline Prostatic & $10(1.2)$ & $10(1.2)$ & $9(1.1)$ \\
\hline Testicular & $1(0.1)$ & 0 & 0 \\
\hline Lung & $6(0.4)$ & $5(0.3)$ & $7(0.5)$ \\
\hline Nasal/laryngeal & $1(0.1)$ & 0 & $2(0.1)$ \\
\hline Melanoma & $2(0.1)$ & $2(0.1)$ & $2(0.1)$ \\
\hline Skin (non-melanoma) & $7(0.5)$ & $5(0.3)$ & $11(0.8)$ \\
\hline Basal cell carcinoma & $6(0.4)$ & $5(0.3)$ & $10(0.7)$ \\
\hline Other & $1(0.1)$ & 0 & $1(0.1)$ \\
\hline Sarcomas & 0 & $1(0.1)$ & 0 \\
\hline
\end{tabular}

analysis) with the same contrast in the RECORD background sulfonylurea stratum. However, ethnic and geographical origin, as well as background therapy differed between the studies. A formal meta-analysis was therefore not performed. Nevertheless, upon combination of the numbers of malignancies in the two studies, the lower 95\% CI would exclude a greater than $17 \%$ (HR 1.07 [95\% CI 0.83-1.39]) advantage of metformin over rosiglitazone.

\section{Discussion}

Based on the analysis of these two randomised controlled trials, metformin is not associated with any demonstrable advantage over rosiglitazone and sulfonylureas in terms of cancer rates, despite total study exposure of nearly 39,000 person-years and study durations of 4 to 6 years. Nevertheless, although the central estimates for hazard ratio of cancer risk for metformin vs rosiglitazone were close to 1.0 in both studies (one higher, the other lower), the data for sulfonylureas are less secure. Thus in ADOPT, the hazard for metformin vs sulfonylureas was $-22 \%$, while in RECORD the hazard for rosiglitazone vs sulfonylureas was $-25 \%$ (neither statistically significant). As others have suggested from observational studies that metformin and sulfonylureas might differ in terms of cancer risk [1, 3], our data can be interpreted as being consistent with that hypothesis, with rosiglitazone being as protective as metformin. It is noteworthy that the study populations are fairly typical of people with type 2 diabetes of moderate duration from diagnosis, approaching a mean age of 60 years at baseline and having mean BMI at entry of around 30 to $33 \mathrm{~kg} / \mathrm{m}^{2}$. Obesity is one of the clearer associations seen with the increased malignancy rate in the diabetes population [12]. The cancer rate of 1.07 patients per 100 person-years in the two studies is consistent with UK national statistics for this 
Table 4 Numbers of participants developing malignancies and selected non-malignant neoplasms in RECORD
Data are number $(n)$ of people $(\%)$ with a neoplasm reported as a serious adverse event

Per cent values for prostate and for breast, cervix/uterine and ovarian cancer are based on men only and women only

Selected benign neoplasms of interest are included

\begin{tabular}{|c|c|c|c|c|}
\hline \multirow[t]{2}{*}{ Variable } & \multicolumn{2}{|c|}{ Background sulfonylurea } & \multicolumn{2}{|c|}{ Background metformin } \\
\hline & Metformin & Rosiglitazone & Sulfonylurea & Rosiglitazone \\
\hline$n$ & 1,122 & 1,103 & 1,105 & 1,117 \\
\hline Study exposure (person-years) & 6,126 & 6,110 & 6,146 & 6,228 \\
\hline \multicolumn{5}{|l|}{ Neoplasms/cancer } \\
\hline All & $81(7.2)$ & $70(6.3)$ & $89(8.1)$ & $73(6.5)$ \\
\hline Malignant neoplasms (non-skin) & $69(6.1)$ & $56(5.1)$ & $74(6.7)$ & $57(5.1)$ \\
\hline Genitourinary & $22(2.0)$ & $23(2.1)$ & $27(2.4)$ & $18(1.6)$ \\
\hline Prostate & $10(1.8)$ & $5(0.9)$ & $11(1.9)$ & $10(1.7)$ \\
\hline Renal & $1(<0.1)$ & $4(0.4)$ & $6(0.5)$ & $3(0.3)$ \\
\hline Uterine & $7(1.3)$ & $7(1.2)$ & $5(1.0)$ & $1(0.2)$ \\
\hline Ovarian & $1(0.2)$ & $3(0.5)$ & $3(0.6)$ & $2(0.4)$ \\
\hline Bladder & $3(0.3)$ & $4(0.4)$ & $2(0.2)$ & $2(0.2)$ \\
\hline Gastrointestinal & $24(2.1)$ & $12(1.1)$ & $21(1.9)$ & $17(1.5)$ \\
\hline Pancreatic & $6(0.5)$ & $1(<0.1)$ & $7(0.6)$ & $1(<0.1)$ \\
\hline Gall bladder/biliary & $3(0.3)$ & 0 & $1(<0.1)$ & $3(0.3)$ \\
\hline Gastric & $1(<0.1)$ & $5(0.5)$ & $2(0.2)$ & $2(0.2)$ \\
\hline Liver & $2(0.2)$ & 0 & $1(<0.1)$ & $1(<0.1)$ \\
\hline Breast & $8(1.4)$ & $4(0.7)$ & $9(1.7)$ & $7(1.4)$ \\
\hline Melanoma & 0 & $2(0.2)$ & $2(0.2)$ & $2(0.2)$ \\
\hline Skin (non-melanoma) & $3(0.3)$ & $8(0.7)$ & $5(0.5)$ & $7(0.6)$ \\
\hline Metastases (unknown primary) & $8(0.7)$ & $4(0.4)$ & $5(0.5)$ & $5(0.4)$ \\
\hline Lung & $3(0.3)$ & $9(0.8)$ & $6(0.5)$ & $3(0.3)$ \\
\hline Haematological & $2(0.2)$ & $2(0.2)$ & $3(0.3)$ & $4(0.4)$ \\
\hline Leukaemia & $1(<0.1)$ & $2(0.2)$ & 0 & $2(0.2)$ \\
\hline Lymphoma & $1(<0.1)$ & 0 & $1(<0.1)$ & $1(<0.1)$ \\
\hline Head and neck & $2(0.2)$ & $1(<0.1)$ & $4(0.4)$ & $1(<0.1)$ \\
\hline Endocrine & $3(0.3)$ & $1(<0.1)$ & $2(0.2)$ & $2(0.2)$ \\
\hline Neurological & 0 & $2(0.2)$ & $2(0.2)$ & $1(<0.1)$ \\
\hline Other & $2(0.2)$ & 0 & $1(<0.1)$ & 0 \\
\hline Benign neoplasms & $12(1.1)$ & $10(0.9)$ & $12(1.1)$ & $10(0.9)$ \\
\hline Colon/rectal polyp & $4(0.4)$ & $1(<0.1)$ & $1(<0.1)$ & 0 \\
\hline Lipoma & 0 & 0 & $1(<0.1)$ & 0 \\
\hline
\end{tabular}

period and age group, allowing that investigators tend not to admit higher risk people to these studies [13].

Although the strength of these data lie in their origin from randomised controlled trials, thus removing the risk of allocation bias that is associated with other types of study, some caution should be exercised when considering the data at face value. For example, these studies were not set up with the primary intention of collecting data on malignancies and so these events were not formally adjudicated. However, as part of the regulatory requirement, a fairly vigorous process for ascertainment of serious adverse events was in place, including regular monitoring of the investigator site. Moreover, even in a blinded study like ADOPT (RECORD being open label), some decisions on whether a neoplasm is malignant or not may have been made open to randomisation drug group and by manufacturer employees, allowing the possibility of conscious or unconscious bias.

Even in large trials such as those considered here, the numbers of cancer events are not large (Tables 1, 2, 3, 4) and differences in other aspects of study conduct can affect interpretation of results. Our results, which come from two independent sources, are fairly secure with regard to the comparison of metformin with rosiglitazone, with central estimates of comparative risk being close to unity and no hint of advantage to metformin. Rosiglitazone, to a greater extent than metformin, is an insulin sensitiser, so both medications may offer common protection against increased malignancy in the obese. Although the numbers of cancer events in metformin and sulfonylurea groups of ADOPT are seemingly similar 
(Table 1), the fewer person-years of exposure to glibenclamide in the sulfonylurea population, a result of the greater number of participants reaching the adverse glucose control endpoint, means that the central estimate for the hazard ratio of malignancies for metformin compared with sulfonylurea is 0.78 (95\% CI 0.53-1.14). This, combined with the observation that in RECORD metformin was similar to rosiglitazone on a background of sulfonylurea, but that rosiglitazone was numerically better than sulfonylureas in the other arm (Table 2), leaves open the possibility that metformin and rosiglitazone may have a small advantage over sulfonylureas.

In RECORD there was some crossover between therapies and use of other therapies such as insulin, as is inevitable in any longer term glucose-control study as islet beta cell dysfunction progresses. The effect of this will be to dilute differences between the studied medications. Nevertheless this crossover only becomes proportionately significant towards the end of the study; as a result total safety exposure to the randomised preparations remained high over the 5.5 years of study (82-88\% of total study exposure in the different arms). Exposure to insulin was also limited as participants needed to reach $\mathrm{HbA}_{1 \mathrm{c}}$ criteria for rescue therapy. As a result, insulin exposure in all groups was $\leq 10 \%$ of study exposure. The per protocol analyses of randomised treatments led to similar conclusions to those from the intention-to-treat population.

It is tempting in RECORD to make comparisons between the two treatment strata, particularly as the cancer rates in participants on rosiglitazone were the same in both populations. However, such comparisons may be unsafe, as the background metformin stratum was clearly more obese and possibly therefore at higher background risk of cancer, although a little younger [9].

Finally our analysis is of two active comparator studies and thus cannot exclude the possibility that all the medications have an absolute advantage or disadvantage for any outcome, either over no medication use or over other therapies. In particular, the issue of comparison of metformin with insulin therapy is not addressed in the present paper, although such data might be available from the UK Prospective Diabetes Study (UKPDS) [14]. In the UKPDS, deaths from cancer were too few for any conclusion to be reached about differences between metformin and conservative management arms (RR 0.71 [95\% CI 0.29-1.76]), while in the main study the cancer death rate was identical in the therapy (sulfonylurea or insulin) and conservative arms (RR 0.98 [95\% CI 0.64 1.52]) $[14,15]$.

We conclude that these studies contain no suggestion that metformin has an advantage over rosiglitazone in terms of protection against malignancy. However, as the numbers of malignancies in the studies are limited, the possibility that sulfonylureas may have a small disadvantage $(\sim<25 \%)$ over the others cannot be excluded by these data. In addition, these data are not useful by themselves with regard to specific malignancies and do not address the question of whether oral glucose-lowering agents as a whole offer protection vs insulin therapy.

Acknowledgements This analysis was unfunded. We are grateful to GlaxoSmithKline (GSK) for access to the database tables and permission to publish information from them. We thank P. Curtis (GSK) for some additional statistical analysis of study participant and medication exposures, and J. Lachin of the ADOPT study group for statistical advice.

Duality of interest The authors or institutions connected with them have received funding from GlaxoSmithKline (GSK) and other oral glucose-lowering drug manufacturers in connection with advisory, educational and research activities, including those relating to rosiglitazone. N. P. Jones and D. Noronha are employees of GSK.

Open Access This article is distributed under the terms of the Creative Commons Attribution Noncommercial License which permits any noncommercial use, distribution, and reproduction in any medium, provided the original author(s) and source are credited.

\section{References}

1. Evans JMM, Donnelly LA, Emslie-Smith AM, Alessi DR, Morris AD (2005) Metformin and reduced risk of cancer in diabetic patients. BMJ 330:1304-1305

2. Bowker SL, Majumdar SR, Veugelers P, Johnson JA (2006) Increased cancer-related mortality for patients with type 2 diabetes who use sulfonylureas or insulin. Diab Care 29:254-258

3. Currie CJ, Poole CD, Gale EAM (2009) The influence of glucoselowering therapies on cancer risk in type 2 diabetes. Diabetologia 52:1766-1777

4. Zakikhani M, Dowling R, Fantus IG et al (2006) Metformin is an AMP kinase-dependent growth inhibitor for breast cancer cells. Cancer Res 66:10269-10273

5. Smith U, Gale EAM (2009) Does diabetes therapy influence the risk of cancer? Diabetologia 52:1699-1708

6. Smith U, Gale EAM (2009) Lantus insulin: a possible link with cancer which requires further investigation. EASD webcast transcript. Available from http://webcast.easd.org/press/glargine/ transcript.htm (accessed 10 August 2009)

7. Holstein A, Nahrwold D, Hinze S, Egberts E-H (1999) Contraindications to metformin therapy are largely disregarded. Diabet Med 16:692-696

8. Kahn SE, Haffner SM, Heise MA et al, for the ADOPT Study Group (2006) Glycemic durability of rosiglitazone, metformin, or glyburide monotherapy. N Engl J Med 355:2427-2443

9. Home PD, Pocock SJ, Beck-Nielsen H et al (2009) Rosiglitazone evaluated for cardiovascular outcomes in oral agent combination therapy for type 2 diabetes (RECORD): a multicentre, randomised, open-label trial. Lancet 373:2125-2135

10. ICH (2009) International Conference on Harmonisation of Technical Requirements for Registration of Pharmaceuticals for Human Use Guidelines. Available from www.ich.org/UrlGrpServer. 
jser?@_ID=276\&@_TEMPLATE=254 (accessed 10 August 2009)

11. Fisher LD, Lin DY (1999) Time-dependent covariates in the Cox proportional-hazards regression model. Ann Rev Public Health $20: 145-157$

12. Giovannucci E, Michaud D (2007) The role of obesity and related metabolic disturbances in cancers of the colon, prostate and pancreas. Gastroenterology 132:2208-2225

13. Office for National Statistics (2004) Registrations of cancer diagnosed in 2004, England. Office for National Statistics, Series MB1:35, London. Available from www.statistics.gov.uk/downloads/
theme_health/MB1_35/MB1_No\%2035_2004.pdf (accessed 10 August 2009).

14. UK Prospective Diabetes Study (UKPDS) Group (1998) Effect of intensive blood-glucose control with metformin on complications in overweight patients with type 2 diabetes (UKPDS 34). Lancet 352:854-865

15. UK Prospective Diabetes Study (UKPDS) Group (1998) Intensive blood-glucose control with sulphonylureas or insulin compared with conventional treatment and risk of complications in patients with type 2 diabetes (UKPDS 33). Lancet 352:837853 